Article

\title{
Coumarins from Edgeworthia chrysantha
}

\author{
Xing-Nuo Li, Sheng-Qiang Tong, Dong-Ping Cheng, Qing-Yong Li * and Ji-Zhong Yan * \\ College of Pharmaceutical Science, Zhejiang University of Technology, 18 Chaowang Road, \\ Hangzhou 310014, China \\ * Authors to whom correspondence should be addressed; E-Mails: liqy@zjut.edu.cn (Q.-Y.L.); \\ yjz@zjut.edu.cn (J.-Z.Y.); Tel.: +86-571-8832-0984 (Q.-Y.L.); +86-571-8832-0506 (J.-Z.Y.); \\ Fax: +86-571-8832-0913 (Q.-Y.L. \& J.-Z.Y.).
}

Received: 9 January 2014; in revised form: 23 January 2014 / Accepted: 23 January 2014 / Published: 13 February 2014

\begin{abstract}
A new coumarin, edgeworic acid (1), was isolated from the flower buds of Edgeworthia chrysantha, together with the five known coumarins umbelliferone (2), 5,7-dimethoxycoumarin (3), daphnoretin (4), edgeworoside C (5), and edgeworoside A (6). Their structures were established on the basis of spectral data, particularly by the use of 1D NMR and several 2D shift-correlated NMR pulse sequences $\left({ }^{1} \mathrm{H}^{-}{ }^{1} \mathrm{H}\right.$ COSY, HSQC and $\mathrm{HMBC}$ ), in combination with acetylation reactions.
\end{abstract}

Keywords: coumarin; Edgeworthia chrysantha; flower buds; edgeworic acid

\section{Introduction}

The genus Edgeworthia (Thymelaeaceae) consists of five species distributed around the World, which are native to China, India, Japan, and southeast of America [1]. E. chrysantha is widely distributed and is endemic to South and East China [1]. The bark of E. chrysantha is used as "Zushima" in some local areas in China for the treatment of traumatic injury and rheumatism [2,3], and the fiber of the stem bark is a raw materials for making high quality paper [1]. The flower buds are often used as the traditional Chinese medicine "Mimenghua" for the treatment of ophthalmalgia and delacrimation $[1,4]$.

Phytochemical studies have revealed that E. chrysantha contains various constituents, such as coumarins [5-8], flavonoids [9-11], terpenes [12-14] and lignans [15,16]. Among them coumarins are generally considered as the major anti-inflammatory and analgesia bioactive constituents [17]. In our 
continuing search for pharmacological and structurally interesting substances from the flower buds of E. chrysantha, a new coumarin, edgeworic acid (1), has been isolated, along with the five known coumarins umbelliferone (2) [18], 5,7-dimethoxycoumarin (3) [19] daphnoretin (4) [20], edgeworoside C (5) [18], and edgeworoside A (6) [18] (Figure 1). The structure of the new compound was elucidated by spectroscopic methods and confirmed by acetylation.

Figure 1. Chemical structures of compounds 1-6.
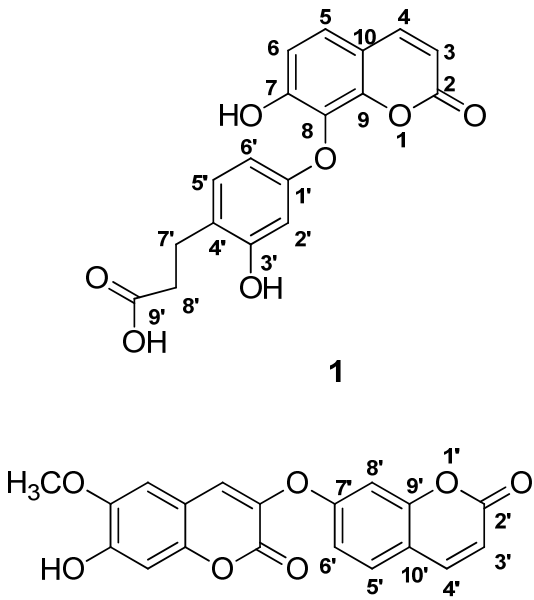

4<smiles>O=c1ccc2ccc(O)cc2o1</smiles>

2

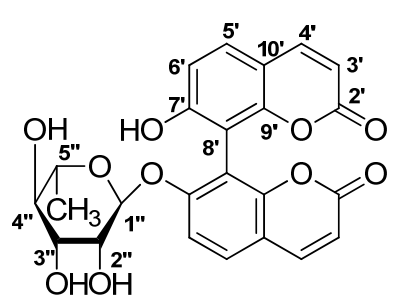

5<smiles>COc1cc(OC)c2ccc(=O)oc2c1</smiles>

3

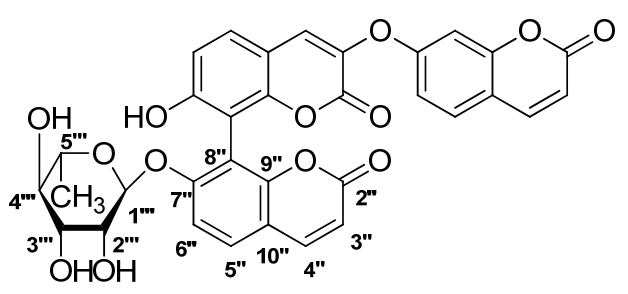

6

\section{Results and Discussion}

Compound 1 was obtained as a white powder. The molecular formula $\mathrm{C}_{18} \mathrm{H}_{14} \mathrm{O}_{7}$ was determined by HR-ESI-MS ([M-H] $]^{-}$peak at $m / z$ 341.0752), indicating 12 degrees of unsaturation. The ${ }^{13} \mathrm{C}-\mathrm{NMR}$ and DEPT spectra resolved 18 carbon signals, which were classified by chemical shifts and HSQC spectrum as two carbonyl groups $\left[\delta_{\mathrm{C}} 174.6\left(\mathrm{C}-9^{\prime}\right), \delta_{\mathrm{C}} 160.2(\mathrm{C}-2)\right]$, seven $s p^{2}$ quaternary carbons $\left[\delta_{\mathrm{C}} 157.2\right.$ $\left(\mathrm{C}-1^{\prime}\right), \delta_{\mathrm{C}} 156.3\left(\mathrm{C}-3^{\prime}\right), \delta_{\mathrm{C}} 154.8(\mathrm{C}-7), \delta_{\mathrm{C}} 148.8(\mathrm{C}-9), \delta_{\mathrm{C}} 128.6(\mathrm{C}-8), \delta_{\mathrm{C}} 121.0\left(\mathrm{C}-4{ }^{\prime}\right), \delta_{\mathrm{C}} 112.5$ $(\mathrm{C}-10)]$, seven $s p^{2}$ methines $\left[\delta_{\mathrm{C}} 145.1(\mathrm{C}-4), \delta_{\mathrm{C}} 130.5(\mathrm{C}-5), \delta_{\mathrm{C}} 125.7(\mathrm{C}-5), \delta_{\mathrm{C}} 114.1(\mathrm{C}-6), \delta_{\mathrm{C}} 112.1\right.$ $(\mathrm{C}-3), \delta_{\mathrm{C}} 105.7\left(\mathrm{C}-6^{\prime}\right), \delta_{\mathrm{C}} 102.0\left(\mathrm{C}-2^{\prime}\right)$ ], two $s p^{3}$ methylenes $\left[\delta_{\mathrm{C}} 34.3\left(\mathrm{C}-8^{\prime}\right), \delta_{\mathrm{C}} 25.3\left(\mathrm{C}-7^{\prime}\right)\right]$ (Table 1).

The IR spectrum exhibited vibration bands for free hydroxyl $\left(3334 \mathrm{~cm}^{-1}\right)$, carboxyl $\left(3207,1732 \mathrm{~cm}^{-1}\right)$, conjugated carbonyl $\left(1692 \mathrm{~cm}^{-1}\right)$, and aromatic $\left(1610,1519,1448 \mathrm{~cm}^{-1}\right)$ functionalities. The UV spectrum exhibited a maximum absorption at $322 \mathrm{~nm}$. According to the data mentioned above, it is suggested that compound $\mathbf{1}$ has a coumarin skeleton. This was further supported by the ${ }^{1} \mathrm{H}-\mathrm{NMR}$ signals $\left[\delta_{\mathrm{H}} 8.01(1 \mathrm{H}, \mathrm{d}, J=9.2 \mathrm{~Hz}, \mathrm{H}-4) ; \delta_{\mathrm{H}} 6.25(1 \mathrm{H}, \mathrm{d}, J=9.2 \mathrm{~Hz}, \mathrm{H}-3)\right]($ Table 1$)$, and ${ }^{13} \mathrm{C}-\mathrm{NMR}$ signals $\left[\delta_{\mathrm{C}} 160.2(\mathrm{C}-2) ; \delta_{\mathrm{C}} 145.1(\mathrm{C}-4) ; \delta_{\mathrm{C}} 112.1(\mathrm{C}-3)\right]$ [8].

The ${ }^{1} \mathrm{H}-\mathrm{NMR}$ spectrum of $\mathbf{1}$ (Table 1) showed the presence of a set of ortho-coupled aromatic signals $\left[\delta_{\mathrm{H}} 7.46(1 \mathrm{H}, \mathrm{d}, J=8.4 \mathrm{~Hz}, \mathrm{H}-5) ; \delta_{\mathrm{H}} 6.99(1 \mathrm{H}, \mathrm{d}, J=8.4 \mathrm{~Hz}, \mathrm{H}-6)\right]$. The ${ }^{1} \mathrm{H}-\mathrm{NMR}$ data also showed an ABX-type coupling system $\left[\delta_{\mathrm{H}} 6.97\left(1 \mathrm{H}, \mathrm{d}, J=8.4 \mathrm{~Hz}, \mathrm{H}-5^{\prime}\right) ; \delta_{\mathrm{H}} 6.29\left(1 \mathrm{H}, \mathrm{d}, J=2.4 \mathrm{~Hz}, \mathrm{H}-2^{\prime}\right)\right.$; $\delta_{\mathrm{H}} 6.24\left(1 \mathrm{H}\right.$, overlapped, $\left.\left.\mathrm{H}-6^{\prime}\right)\right]$. The signals at $\delta_{\mathrm{H}} 2.68\left(2 \mathrm{H}, \mathrm{t}, J=7.2 \mathrm{~Hz}, \mathrm{H}-7^{\prime}\right), \delta_{\mathrm{C}} 25.3\left(\mathrm{C}-7^{\prime}\right), \delta_{\mathrm{H}} 2.43$ $\left(2 \mathrm{H}, \mathrm{t}, J=7.2 \mathrm{~Hz}, \mathrm{H}-8^{\prime}\right), \delta_{\mathrm{C}} 34.3\left(\mathrm{C}-8^{\prime}\right)$ showed the existence of a 3-propionic acid group [8], which was confirmed by the HSQC, HMBC, and ${ }^{1} \mathrm{H}-{ }^{1} \mathrm{H}$ COSY spectra. (Figure 2) In the HMBC spectrum, the ${ }^{1} \mathrm{H}-\mathrm{NMR}$ signal at $\delta_{\mathrm{H}} 2.43\left(\mathrm{H}-8^{\prime}\right)$ was correlated to ${ }^{13} \mathrm{C}-\mathrm{NMR}$ signal at $\delta_{\mathrm{C}} 121.0\left(\mathrm{C}-4^{\prime}\right)$, and the 
${ }^{1} \mathrm{H}-\mathrm{NMR}$ signal at $\delta_{\mathrm{H}} 2.68\left(\mathrm{H}^{-} 7^{\prime}\right)$ showed correlations with ${ }^{13} \mathrm{C}-\mathrm{NMR}$ signals at $\delta_{\mathrm{C}} 156.3\left(\mathrm{C}-3^{\prime}\right), 121.0$ (C-4') and 130.5 (C-5'), indicating that 3-propionic acid group was located at the C-4' position. (Figure 2) The aromatic $\mathrm{H}$-atom at $\delta_{\mathrm{H}} 7.46$, which correlated with 154.8 (C-7), 148.8 (C-9), 145.1 (C-4) in the HMBC spectrum (Figure 2), could be assigned to H-5. Since it coupled with the H-6, the substitution site at the coumarin skeleton was established at C-7 and C-8.

Table 1. ${ }^{1} \mathrm{H}$ - and ${ }^{13} \mathrm{C}-\mathrm{NMR}$ data of $\mathbf{1}$ and $\mathbf{1 a}(\delta$ in ppm and $J$ in $\mathrm{Hz})$.

\begin{tabular}{cccccc}
\hline & & $\mathbf{1}$ & & & \multicolumn{2}{c}{$\mathbf{1 a}$} \\
\cline { 2 - 3 } \cline { 5 - 6 } No. No. & $\boldsymbol{\delta}_{\mathbf{H}}$ & $\boldsymbol{\delta}_{\mathbf{C}}$ & & $\boldsymbol{\delta}_{\mathbf{H}}$ & $\boldsymbol{\delta}_{\mathbf{C}}$ \\
\hline 2 & - & $160.2 \mathrm{~s}$ & 2 & - & $159.3 \mathrm{~s}$ \\
3 & $6.25(\mathrm{~d}, 9.2)$ & $112.1 \mathrm{~d}$ & 3 & $6.53(\mathrm{~d}, 9.6)$ & $116.5 \mathrm{~d}$ \\
4 & $8.01(\mathrm{~d}, 9.2)$ & $145.1 \mathrm{~d}$ & 4 & $8.13(\mathrm{~d}, 9.6)$ & $144.4 \mathrm{~d}$ \\
5 & $7.46(\mathrm{~d}, 8.4)$ & $125.7 \mathrm{~d}$ & 5 & $7.71(\mathrm{~d}, 8.4)$ & $125.6 \mathrm{~d}$ \\
6 & $6.99(\mathrm{~d}, 8.4)$ & $114.1 \mathrm{~d}$ & 6 & $7.33(\mathrm{~d}, 8.4)$ & $120.3 \mathrm{~d}$ \\
7 & - & $154.8 \mathrm{~s}$ & 7 & - & $146.1 \mathrm{~s}$ \\
8 & - & $128.6 \mathrm{~s}$ & 8 & - & $133.3 \mathrm{~s}$ \\
9 & - & $148.8 \mathrm{~s}$ & 9 & - & $147.7 \mathrm{~s}$ \\
10 & - & $112.5 \mathrm{~s}$ & 10 & - & $118.9 \mathrm{~s}$ \\
$1^{\prime}$ & - & $157.2 \mathrm{~s}$ & $1^{\prime}$ & - & $157.0 \mathrm{~s}$ \\
$2^{\prime}$ & $6.29(\mathrm{~d}, 2.4)$ & $102.0 \mathrm{~d}$ & $2^{\prime}$ & $6.65(\mathrm{~d}, 2.4)$ & $104.0 \mathrm{~d}$ \\
$3^{\prime}$ & - & $156.3 \mathrm{~s}$ & $3^{\prime}$ & - & $152.7 \mathrm{~s}$ \\
$4^{\prime}$ & - & $121.0 \mathrm{~s}$ & $4^{\prime}$ & - & $118.1 \mathrm{~s}$ \\
$5^{\prime}$ & $6.97(\mathrm{~d}, 8.4)$ & $130.5 \mathrm{~d}$ & $5^{\prime}$ & $7.24(\mathrm{~d}, 8.4)$ & $129.6 \mathrm{~d}$ \\
$6^{\prime}$ & $6.24($ overlapped) & $105.7 \mathrm{~d}$ & $6^{\prime}$ & $6.69(\mathrm{dd}, 2.4,8.4)$ & $111.2 \mathrm{~d}$ \\
$7^{\prime}$ & $2.68(\mathrm{t}, 7.2)$ & $25.3 \mathrm{t}$ & $7^{\prime}$ & $2.94(\mathrm{t}, 7.2)$ & $22.5 \mathrm{t}$ \\
$8^{\prime}$ & $2.43(\mathrm{t}, 7.2)$ & $34.3 \mathrm{t}$ & $8^{\prime}$ & $2.78(\mathrm{t}, 7.2)$ & $28.9 \mathrm{t}$ \\
$9^{\prime}$ & - & $174.6 \mathrm{~s}$ & $9^{\prime}$ & - & $168.4 \mathrm{~s}$ \\
& & & & & \\
& & & Ac & $2.15(\mathrm{~s})$ & $20.6 \mathrm{q}$ \\
& & & & & $168.5 \mathrm{~s}$ \\
\hline
\end{tabular}

Figure 2. Key HMBC and ${ }^{1} \mathrm{H}-{ }^{1} \mathrm{H}$ COSY correlations of $\mathbf{1}$ and $\mathbf{1 a}$.

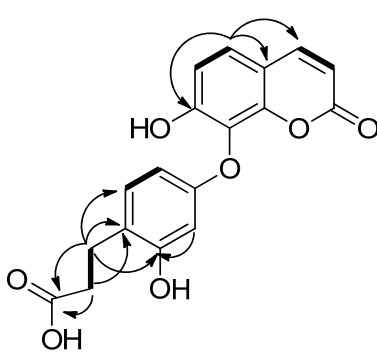

1

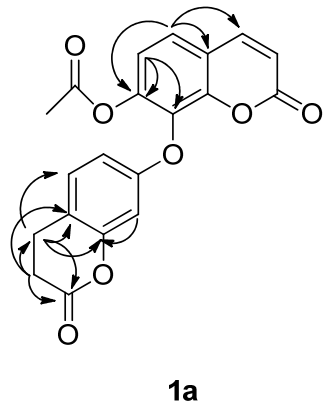

$1 \mathbf{a}$

\section{$\overbrace{\mathrm{HMBC}}^{\cos Y}$}

According to the molecular formula of 1, there were only two phenolic hydroxyl groups left. Unfortunately, no phenolic hydroxyl groups signals appeared in the ${ }^{1} \mathrm{H}-\mathrm{NMR}$ spectrum, so the locations of the phenolic hydroxyl groups were confirmed by acetylation. The acetylated derivative of 
1 (compound 1a) was obtained as a white powder. The molecular formula $\mathrm{C}_{20} \mathrm{H}_{16} \mathrm{O}_{7}$ was determined by ESI-MS $\left([\mathrm{M}+\mathrm{H}]^{+}\right.$peak at $m / z$ 367.0), indicating 13 degrees of unsaturation. By comparing the MS data of $1 \mathrm{a}$ with those of $\mathbf{1}$, it was presumed that a six membered lactone ring was formed during the acetylation reaction. The comparison of the ${ }^{13} \mathrm{C}-\mathrm{NMR}$ data of $\mathbf{1}$ with those of $\mathbf{1 a}$ (Table 1) revealed that the signals of C- 6 and C-8 were shifted downfield in the range of $\delta 5-6 \mathrm{ppm}$, the signal of C-7 was shifted upfield by $\delta 8.7 \mathrm{ppm}$, and the signal of C-3' was shifted upfield by $\delta 3.6 \mathrm{ppm}$. According to the analysis mentioned above, one of the phenolic hydroxyl groups was deduced to be at C-7 [21], and another one was located at C-3', which was confirmed by the HMBC spectrum. (Figure 2).

The five known coumarins were identified as umbelliferone (2) [18], 5,7-dimethoxycoumarin (3) [19] daphnoretin (4) [20], edgeworoside C (5) [18], and edgeworoside A (6) [18], by interpretation of their spectroscopic data and comparison with literature values.

\section{Experimental}

\subsection{General}

All chemical solvents used were of analytical grade. Column chromatography (CC): MCI gel (Mitsubishi Chemical Co., Tokyo, Japan); Sephadex LH-20 (Pharmacia Fine Chemical Co. Ltd., Uppsala, Sweden); silica gel (Qingdao Marine Chemical Group Co., Qingdao, China; 200-300 and 400-600 mesh). HPLC: Agilent 1100 series (Agilent Technologies, Palo Alto, CA, USA) equipped with an Agilent DAD spectrophotometer and an Alltima-C18 reversed-phase column $(5 \mu \mathrm{m}, 250 \times 10 \mathrm{~mm})$ with an Eclipse XDB-C18 guard column. IR spectra: Nicolet-Magna-FT-IR 750 spectrometer (Thermo Scientific, Waltham, MA, USA). UV spectra: Shimadzu UV-2450 spectrophotometer (Shimadzu, Kyoto, Japan). LR- and HR-ESI-MS: Finnigan LCQ-Deca (Thermo Scientific, Waltham, MA, USA) and Waters Micromass Q-TOF-Ultima mass spectrometers (Waters, Milford, MA, USA).

${ }^{1} \mathrm{H}$ - and ${ }^{13} \mathrm{C}-\mathrm{NMR}$ spectra were recorded on a Bruker Avance-400 spectrometer (Bruker, Karlsruhe, Germany) $\left({ }^{1} \mathrm{H}\right.$ - at $400 \mathrm{MHz},{ }^{13} \mathrm{C}$ - at $\left.100 \mathrm{MHz}\right)$ in DMSO- $d_{6}$ at room temperature $\left(22{ }^{\circ} \mathrm{C}\right)$. Chemical shifts are reported in ppm $(\delta)$, relative to tetramethylsilane as internal standard, and coupling constants are in Hertz.

\subsection{Plant Material}

The flower buds of E. chrysantha were collected in a garden of Lishui, Zhejiang Province, China, in February 2008. The plants were authenticated by Dr. Chu Chu, Zhejiang University of Technology, China. A voucher specimen (TCM 2008-026) was deposited in College of Pharmaceutical Science, Zhejiang University of Technology.

\subsection{Extraction and Isolation}

The air-dried material $(10 \mathrm{~kg})$ were extracted at room temperature and for $36 \mathrm{~h} \times 3$ with $95 \%(v / v)$ $\mathrm{EtOH}(5 \mathrm{~L} \times 3)$ to give, after removal of the solvent, $220 \mathrm{~g}$ of crude extract which was dissolved in $4 \mathrm{~L}$ of $\mathrm{H}_{2} \mathrm{O}$ to form a suspension and successively partitioned with petroleum ether $\left(60-90{ }^{\circ} \mathrm{C}\right)(3,000 \mathrm{~mL} \times 3)$, ethyl acetate $(3,000 \mathrm{~mL} \times 3)$ and $n$-butanol $(3,000 \mathrm{~mL} \times 3)$. The ethyl acetate extracts $(14 \mathrm{~g})$ were chromatographed on a silica gel column (petroleum ether/ethyl acetate, 4:1-0:1 v/v) to give eight 
fractions $1-8$. Fraction $1(4: 1 v / v ; 1.2 \mathrm{~g})$ was separated on a silica gel $\mathrm{H}$ column (petroleum ether/ethyl acetate, $6: 1 \mathrm{v} / \mathrm{v})$ to afford three fractions 1'-3'. Subfraction 3' (100 mg) was chromatographed on a silica gel H column (petroleum ether/acetone, 6:1 v/v) to afford compound 2 (28 mg) and $\mathbf{3}(27 \mathrm{mg})$. Fraction $3(3: 1 v / v ; 0.2 \mathrm{~g})$ was recrystallized with methanol to give compound $4(45 \mathrm{mg})$. Fraction $4(2.5: 1 v / v$; $1.0 \mathrm{~g})$ was separated on a silica gel $\mathrm{H}$ column $\left(\mathrm{CHCl}_{3} /\right.$ acetone, $\left.6: 1 \mathrm{v} / \mathrm{v}\right)$ to afford compound 1 (43 $\left.\mathrm{mg}\right)$. Fraction $7(1: 1 v / v ; 3.8 \mathrm{~g})$ was chromatographed on a $\mathrm{MCI}$ gel column $\left(\mathrm{MeOH} / \mathrm{H}_{2} \mathrm{O}, 1: 9-8: 2 v / v\right)$ to give three fractions 1'-3'. Subfraction 1' (100 mg) was recrystallized from methanol to afford compound 5 (55 mg). Subfraction 3' (80 mg) was subjected to Sephadex LH-20 column chromatography $(3 \times 100 \mathrm{~cm})$ eluted with $\mathrm{CHCl}_{3} / \mathrm{MeOH}(1: 1 \mathrm{v} / \mathrm{v})$ to remove the pigments and finally purified by semipreparative-HPLC using $\mathrm{MeOH} / \mathrm{H}_{2} \mathrm{O}\left(64: 36 v / v, 25^{\circ} \mathrm{C}, 3.0 \mathrm{ml} / \mathrm{min}\right)$ to afford compound $6\left(13 \mathrm{mg}, \mathrm{t}_{\mathrm{R}}=17.38 \mathrm{~min}\right)$.

\subsection{Acetylation of Edgeworic acid (1)}

A mixture of compound $1(20 \mathrm{mg}), \mathrm{Ac}_{2} \mathrm{O}(5 \mathrm{~mL})$, and pyridine $(5 \mathrm{~mL})$ was stirred at room temperature overnight. The resulting solution was concentrated under vacuum. The residue was dissolved in ethyl acetate and washed with water $(5 \mathrm{~mL})$. The product was purified on a silica gel column ( $n$-hexane/ethyl acetate, $3: 2 \mathrm{v} / \mathrm{v})$ to afford $\mathbf{1 a}(15 \mathrm{mg})$.

Acetylated derivative of edgeworic acid (1a). White powder; ESI-MS (+) $\mathrm{m} / z \quad 367.0 \quad[\mathrm{M}+\mathrm{H}]^{+}$; ${ }^{1} \mathrm{H}-\mathrm{NMR}$ (DMSO- $d_{6}$ ) and ${ }^{13} \mathrm{C}-\mathrm{NMR}$ (DMSO- $d_{6}$ ) data: see Table 1.

\subsection{Spectral Data}

Edgeworic acid (1). White powder; UV (MeOH) $\lambda_{\max }(\log \varepsilon): 322(4.20)$; IR (KBr) $v_{\max }: 3,334,3,207$, 1732, 1692, 1610, 1519, $1448 \mathrm{~cm}^{-1}$; HR-ESI-MS (-) m/z $341.0752[\mathrm{M}-\mathrm{H}]^{-}$(calcd. for $\mathrm{C}_{18} \mathrm{H}_{13} \mathrm{O}_{7}$, 341.0661); ${ }^{1} \mathrm{H}-\mathrm{NMR}$ (DMSO- $d_{6}$ ) and ${ }^{13} \mathrm{C}-\mathrm{NMR}$ (DMSO- $d_{6}$ ) data: see Table 1.

Umbelliferone (2). Colorless needles; m.p.: 225-228 ${ }^{\circ} \mathrm{C} ;{ }^{1} \mathrm{H}-\mathrm{NMR}$ (DMSO- $\left.d_{6}\right) \delta$ : 10.6 (1H, s, 7-OH), $7.92(1 \mathrm{H}, \mathrm{d}, J=9.5 \mathrm{~Hz}, \mathrm{H}-4), 7.52(1 \mathrm{H}, \mathrm{d}, J=8.5 \mathrm{~Hz}, \mathrm{H}-5), 6.78(1 \mathrm{H}, \mathrm{dd}, J=2.4,8.5 \mathrm{~Hz}, \mathrm{H}-6), 6.71$ $(1 \mathrm{H}, \mathrm{d}, J=2.4 \mathrm{~Hz}, \mathrm{H}-8), 6.19$ (1H, d, $J=9.5 \mathrm{~Hz}, \mathrm{H}-3)$.

5,7-Dimethoxycoumarin (3). Colorless needles; m.p.: $144-145{ }^{\circ} \mathrm{C} ;{ }^{1} \mathrm{H}-\mathrm{NMR}$ (DMSO- $\left.d_{6}\right) \delta: 7.96(1 \mathrm{H}$, d, $J=9.6 \mathrm{~Hz}, \mathrm{H}-4), 6.41(1 \mathrm{H}, \mathrm{s}, \mathrm{H}-8), 6.28(1 \mathrm{H}, \mathrm{s}, \mathrm{H}-6), 6.15(1 \mathrm{H}, \mathrm{d}, J=9.6 \mathrm{~Hz}, \mathrm{H}-3), 3.89(3 \mathrm{H}, \mathrm{s}$, $\left.\mathrm{OCH}_{3}\right), 3.86\left(3 \mathrm{H}, \mathrm{s}, \mathrm{OCH}_{3}\right)$.

Daphnoretin (4). Yellow needles; m.p.: $223-225{ }^{\circ} \mathrm{C} ;{ }^{1} \mathrm{H}-\mathrm{NMR}$ (DMSO- $\left.d_{6}\right) \delta$ : $10.3(1 \mathrm{H}, \mathrm{s}, 7-\mathrm{OH}), 8.05(1 \mathrm{H}$, d, $\left.J=9.5 \mathrm{~Hz}, \mathrm{H}-4^{\prime}\right), 7.88$ (1H, s, H-4), $7.72\left(1 \mathrm{H}, \mathrm{d}, J=8.6 \mathrm{~Hz}, \mathrm{H}-55^{\prime}\right), 7.22(1 \mathrm{H}, \mathrm{s}, \mathrm{H}-5), 7.20$ (1H, d, $\left.J=2.4 \mathrm{~Hz}, \mathrm{H}-8^{\prime}\right), 7.12\left(1 \mathrm{H}, \mathrm{dd}, J=8.6,2.4 \mathrm{~Hz}, \mathrm{H}-6^{\prime}\right), 6.87$ (1H, s, H-8), 6.39 (1H, d, $\left.J=9.5 \mathrm{~Hz}, \mathrm{H}-3^{\prime}\right)$, $3.82\left(3 \mathrm{H}, \mathrm{s}, 6-\mathrm{OCH}_{3}\right) ;{ }^{13} \mathrm{C}-\mathrm{NMR}\left(\mathrm{DMSO}-d_{6}\right) \delta: 160.4(\mathrm{C}-2), 160.1\left(\mathrm{C}-2^{\prime}\right), 157.4\left(\mathrm{C}-7^{\prime}\right), 155.5\left(\mathrm{C}-9^{\prime}\right)$, 150.8 (C-7), 147.9 (C-9), 146.1 (C-6), 144.5 (C-4'), 136.2 (C-3), 131.2 (C-4), 130.3 (C-5'), 114.9 (C-10'), 114.3 (C-3'), 113.9 (C-6'), 110.6 (C-10), 110.0 (C-5), 104.5 (C-8'), 103.2 (C-8), 56.5 (7-OCH $)_{3}$. 
Edgeworoside $C(\mathbf{5})$. White powder; ${ }^{1} \mathrm{H}-\mathrm{NMR}\left(\mathrm{DMSO}-d_{6}\right) \delta: 10.6(1 \mathrm{H}$, br s, 7'-OH), $8.09(1 \mathrm{H}, \mathrm{d}$, $\left.J=9.2 \mathrm{~Hz}, \mathrm{H}-4^{\prime}\right), 8.03(1 \mathrm{H}, \mathrm{d}, J=9.4 \mathrm{~Hz}, \mathrm{H}-4), 7.78\left(1 \mathrm{H}, \mathrm{d}, J=8.5 \mathrm{~Hz}, \mathrm{H}-5{ }^{\prime}\right), 7.64(1 \mathrm{H}, \mathrm{d}, J=8.3 \mathrm{~Hz}$, H-5), 7.32 (1H, d, $\left.J=8.5 \mathrm{~Hz}, \mathrm{H}-6^{\prime}\right), 7.00$ (1H, d, $\left.J=8.3 \mathrm{~Hz}, \mathrm{H}-6\right), 6.33$ (1H, d, $\left.J=9.2 \mathrm{~Hz}, \mathrm{H}-3^{\prime}\right), 6.21$ $(1 \mathrm{H}, \mathrm{d}, J=9.4 \mathrm{~Hz}, \mathrm{H}-3), 5.48$ (1H, s, H-1"), 3.44 (1H, s, H-2"), 3.18 (2H, m, H-4", 5"), 3.01 (1H, br s, H-3"), $1.06\left(3 \mathrm{H}, \mathrm{d}, J=6.0 \mathrm{~Hz}, 5 "-\mathrm{CH}_{3}\right) ;{ }^{13} \mathrm{C}$ NMR (DMSO- $\left.d_{6}\right) \delta: 160.7$ (C-2), 160.5 (C-2'), 159.7 (C-7), 157.5 (C-7'), 153.6 (C-9), 153.1 (C-9'), 145.4 (C-4'), 145.1 (C-4), 129.8 (C-5, 5'), 113.9 (C-10'), 113.4 (C-3'), 113.1 (C-6), 111.8 (C-6'), 111.6 (C-10), 111.5 (C-3), 110.4 (C-8'), 106.9 (C-8), 99.0 (C-1"), 71.9 (C-4"), 70.6 (C-3"), 70.4 (C-2"), 70.1 (C-5"), 18.3 (C-6").

Edgeworoside $A(\mathbf{6})$. White powder; ${ }^{1} \mathrm{H}-\mathrm{NMR}$ (DMSO- $\left.d_{6}\right) \delta: 10.5(1 \mathrm{H}, \mathrm{br}$ s, $7-\mathrm{OH}), 8.10(1 \mathrm{H}, \mathrm{d}$, $\left.J=9.6 \mathrm{~Hz}, \mathrm{H}-4^{\prime \prime}\right), 8.02$ (1H, s, H-4), 8.01 (1H, d, $\left.J=9.5 \mathrm{~Hz}, \mathrm{H}-4{ }^{\prime}\right), 7.80$ (1H, d, $\left.J=8.9 \mathrm{~Hz}, \mathrm{H}-5 "\right), 7.69$ $\left(1 \mathrm{H}, \mathrm{d}, J=8.50 \mathrm{~Hz}, \mathrm{H}-5^{\prime}\right), 7.64(1 \mathrm{H}, \mathrm{d}, J=8.6 \mathrm{~Hz}, \mathrm{H}-5), 7.32(1 \mathrm{H}, \mathrm{d}, J=8.9 \mathrm{~Hz}, \mathrm{H}-6$ ' '), 7.18 (1H, d, $\left.J=2.2 \mathrm{~Hz}, \mathrm{H}-8^{\prime}\right), 7.07\left(1 \mathrm{H}, \mathrm{dd}, J=8.5,2.2 \mathrm{~Hz}, \mathrm{H}-6^{\prime}\right), 7.06(1 \mathrm{H}, \mathrm{d}, J=8.6 \mathrm{~Hz}, \mathrm{H}-6), 6.37(1 \mathrm{H}, \mathrm{d}$, $\left.J=9.5 \mathrm{~Hz}, \mathrm{H}-3^{\prime}\right), 6.34$ (1H, d, $\left.J=9.6 \mathrm{~Hz}, \mathrm{H}-3 "\right), 5.50$ (1H, br s, H-1"'), 3.54 (1H, m, H-2"'), 3.29 (1H, m, H-5"'), 3.19 (1H, m, H-4'"), 2.94 (1H, m, H-3"'), 1.04 (3H, d, $J=6.2$ Hz, 5"'-CH 3 ).

\section{Conclusions}

A new coumarin, edgeworic acid (1), was isolated from the flower buds of $E$. chrysantha together with the five known compounds umbelliferone (2), 5,7-dimethoxycoumarin (3), daphnoretin (4), edgeworoside $\mathrm{C}(\mathbf{5})$, and edgeworoside $\mathrm{A}(\mathbf{6})$. Their structures were determined by spectroscopic analysis 1D-NMR, 2D-NMR and MS experiment combined with an acetylation reaction.

\section{Acknowledgments}

The authors gratefully acknowledge financial support from the National Natural Science Foundation of China (No. 31270397) and Science and Technology Department of Zhejiang Province (2012C23112).

\section{Conflicts of Interest}

The authors declare no conflict of interest.

\section{References}

1. Editorial Group of Flora Republicae Popularis Sinicae. Thymelaeaceae. In Flora Republicae Popularis Sinicae; Gu, C.Z., Li, Z.Y., Eds.; Science Press: Beijing, China, 1999; Volume 52, pp. 389-391.

2. Li, S.H.; Wu, L.J.; Yin, H.Y. Chemical and pharmacological advances of the study on Zushima. J. Chin. Mater. Med. 2002, 27, 401-403.

3. Jiangsu New Medical College. Dictionary of Chinese Crude Drugs; Shanghai People' Publishing House: Shanghai, China, 1977; Volume 2, p. 1739.

4. Jiangsu New Medical College. Dictionary of Chinese Crude Drugs; Shanghai People' Publishing House: Shanghai, China, 1977; Volume 2, p. 2264. 
5. Baba, K.; Tabata, Y.; Taniguti, M.; Kozawa, M. Coumarins from Edgeworthia chrysantha. Phytochemistry 1989, 28, 221-225.

6. Baba, K.; Taniguti, M.; Yoneda, Y.; Kozawa, M. Coumarin glycosides from Edgeworthia chrysantha. Phytochemistry 1990, 29, 247-249.

7. Li, S.H.; Wu, L.J.; Gao, H.Y.; Chen, Y.H.; Li, Y. A new dicoumarinoid glycoside from Daphne giraldii. J. Asian Nat. Prod. Res. 2005, 7, 839-842.

8. Hu, X.J.; Jin, H.Z.; Zhang, W.D.; Zhang, W.; Yan, S.K.; Liu, R.H.; Shen, Y.H.; Xu, W.Z. Two new coumarins from Edgeworthia chrysantha. Nat. Prod. Res. 2009, 23, 1259-1264.

9. Zhang, H.J.; Zhao, Y.Y.; Li, O.Y. Studies on the chemical constituents from the flowers of Edgeworthia chrysantha. Nat. Prod. Res. Dev. 1997, 9, 24-27.

10. Zhou, G.X.; Yang, Y.C.; Shi, J.G.; Hu, W.Y. Study on biflavonoids from stem bark of Daphne giraldii. Chin. Tradit. Herb. Drugs. 2002, 33, 1061-1063.

11. Zhou, T.; Zhang, S.W.; Liu, S.S.; Cong, H.J.; Xuan, L.J. Daphnodorin dimers from Edgeworthia chrysantha with $\alpha$-glucosidase inhibitory activity. Phytochem. Lett. 2010, 3, 242-247.

12. Ohigashi, H.; Hirota, M.; Ohtsuka, R.; Koshimizu, K.; Tokuds, H.; Tennen, Y. Plant constituents with Epstein-Barr virus inducing activity: Tigliane and 1-alkyl-daphnane type esters. Symp. Chem. Nat. Prod. 1983, 26, 24-31.

13. Wang, C.R.; An, B.Z.; Li, S.M.; Zhou, B.N. The studies on the bioactive diterpenes from Daphne giraldii. Acta Chim. Sin. 1987, 45, 993-996.

14. Liao, S.G.; Zhang, B.L.; Wu, Y.; Yue, J.M. New phenolic components from Daphne giraldii. Helv. Chim. Acta 2005, 88, 2873-2878.

15. Zhuang, L.G.; Seligmann, O.; Jurcic, K.; Wagner, H. Constituents of Daphne tangutica. Planta Med. 1982, 45, 172-176.

16. Zhuang, L.G.; Seligmann, O.; Wagner, H. Daphneticin, a coumarinolignoid from Daphne tangutica. Phytochemistry 1983, 22, 617-619.

17. Zhang, W.; Su, J.; Hu, X.J.; Liu, R.H.; Zhang, W.D. Chemical constituents and pharmacological activities of three origin plants of Traditional Chinese Medicine Zushima. Chin. J. Pharm. 2007, $38,233-238$.

18. Hu, X.J.; Jin, H.Z.; Su, J.; Zhang, W.; Xu, W.Z.; Yan, S.K.; Liu, R.H.; Li, J.Q.; Zhang, W.D. Coumarins from Daphne retusa. Chin. J. Nat. Med. 2009, 7, 34-36.

19. Yin, F.; Lou, F.C. Studies on the constituents of Citrus medica L. var. Sarcodactylis. Chin. Pharm. J. 2004, 39, 20-21.

20. Chen, Y.Y.; Duan, J.A.; Tang, Y.P.; Guo, S. Chemical constituents from flower buds of Daphne genkwa. Chin. Tradit. Herb. Drugs 2013, 44, 397-402.

21. Wu, L.J.; Qiu, F.; Kong, L.Y.; Liu, Y.X.; Song, S.J.; Lou, H.X. Spectrometric Identification of Organic Compounds, 3rd ed.; China Medical Science Press: Beijing, China, 2009; pp. 167-168.

Sample Availability: Not available.

(C) 2014 by the authors; licensee MDPI, Basel, Switzerland. This article is an open access article distributed under the terms and conditions of the Creative Commons Attribution license (http://creativecommons.org/licenses/by/3.0/). 